\title{
Anatomical Asymmetry of Human Breast for Indicator of Breast Cancer
}

\author{
Prof. Samir Kumar Bandyopadhyay \\ Professor \\ Dept. of Computer Science \& Engineering \\ University of Calcutta, \\ 92 A.P.C. Road, Kolkata - 700009
}

\author{
Indra Kanta Maitra \\ Research Scholar \\ Dept. of Computer Science \& Engineering \\ University of Calcutta, \\ 92 A.P.C. Road, Kolkata - 700009
}

\begin{abstract}
Various subjects that are paired usually are not identically the same, asymmetry is perfectly normal but sometimes asymmetry can be noticeable too much. Breast asymmetry is one of such examples, which is a difference in breast size or shape, or both. Asymmetry analysis of beast has great importance because it is not only indicator for breast cancer but also predict future potential risk for the same. In our research work, we have concentrated to segment the anatomical regions of breast, isolate the border line of each to investigate the presence of abnormal mass and asymmetry of anatomical regions in a pair of mammogram. We used three techniques i.e. contrast enhancement, binary homogeneity enhancement with uniform color reduction and seeded region growing algorithm for the same. The proposed technique, we have obtained $90 \%$ of near accurate result including accurate results on selected 50 numbers different mammograms of MIAS Database. To summarize, the results obtained by the method show that it is a robust approach but it can be improved in terms of accuracy.
\end{abstract}

\section{Keywords}

Mammogram, MIAS Database, CLAHE, BHEA, Seeded Region Growing.

\section{INTRODUCTION}

Some degree of asymmetry is perfectly normal. Whether it's the size of ears to the length of toes, there will always be some variation. Perfect symmetry in human bodies is disturbed by a number of factors, including the secretion of hormones etc.

Most commonly the left breast is different from the right in size and shape. Breast asymmetry usually occurs due to either genetic tendencies or random events in which paired organs like the breasts just grow differentially with respect to starting and stopping. The breasts are stimulated to grow under the influence of estrogen hormone in young girls. It is during the time of growth that is same or very similar size or shape. It is not likely that the size and shape of the breasts will change until pregnancy or the beginning of menopause.

\section{BREAST ASYMMETRY AND BREAST CANCER}

However, sometimes asymmetry can be noticeable too much. The study looked at all asymptomatic women who had self-referred for a mammogram at Liverpool Breast Screening Unit between 1979 and 1986. Breast volume is measured and calculated from the mammograms of all the women. However, the study does provide the first direct evidence that breast asymmetry is linked to breast cancer. Asymmetrical breasts could prove to be reliable indicators of future breast disease in women and this factor should be considered in a woman's risk profile [1]. Breast cancer is the most common malignancy among women and it is the most common cause of cancer death in women [2].

In our research work, we have concentrated to segment the anatomical regions of breast like lobules, fatty tissues, pectoral muscle, ducts etc. the boarder line of each are isolated by which the presence of abnormal mass and asymmetry of anatomical regions are identified in a pair of mammogram. Percentage of asymmetry can be obtained by comparing the regions. Asymmetry analysis is very important not only identification of diseases but to predict future risk, cosmetic and reconstruction surgery.

\section{PREVIOUS WORKS}

Georgsson (2003) proposed method for bilateral registration based on anatomical features and assumptions of how the female breast is deformed under compression. Matches the bilateral counterpart in size and transformed the skin lines of the two breasts to match each other [3].

Good et al. (2003) applied techniques for automatically making the appropriate local image corrections, based on the variations in tissue thickness during breast compression [4].

Méndez et al. (1998) also used nipple and breast border as a reference point for the alignment of the breasts. The coordinates of the detected nipples of both images determined the displacement and the coordinates of the points along the detected breast borders were used to determine the angle of rotation to achieve correspondence [5].

Yin et al. (1994) extracted breast border and nipple position as landmarks for the alignment of left and right breast. They identified breast border by a four-point connectivity tracker and nipple position was identified based on the presence of a thicker skin line and greater subcutaneous parenchymal opacity around the nipple position [6].

\section{SEGMENTATION AND SEEDED REGION GROWING}

The paper is based on the image segmentation method, which refers to the major step in image processing, the inputs are images 
and, outputs are the attributes extracted from those images [7]. The goal of segmentation is to isolate the regions of interest depending on the problem and its characters. A gray level image consists of two main features, namely region and edge. Segmentation algorithms for gray images are generally based on of two basic properties of image intensity values, discontinuity and similarity.

For the segmentation of intensity images like digital mammogram, there are four main approaches [8][9]. Region-based methods rely on the postulate that neighboring pixels within the one region have similar value. This leads to the class of algorithms known as region growing of which the "split and merge" technique [10] is probably the best known.

We used a method "seeded region growing" (SRG), which seed or a set of seed can be automatically or manually selected. Their automated selection can be based on finding pixels that are of interest. The method is employed to segment an image into different regions using a set of seeds. The method has the advantage that it is fairly robust, quick, and parameter free except for its dependency on the order of pixel processing.

\section{PROPOSED METHOD}

Digital Mammograms are medical images that are difficult to interpret, thus a preparation phase is needed in order to improve the image quality and make the segmentation results more accurate i.e. by removing the irrelevant and unwanted parts in the background of the mammogram.

\subsection{Contrast Enhancement of Digital Mammogram}

The contrast enhancement phase is done using the Contrast Limited Adaptive Histogram Equalization (CLAHE) technique, which is a special case of the histogram equalization technique [11] that functions adaptively on the image to be enhanced. The CLAHE method seeks to reduce the noise and edge-shadowing effect produced in homogeneous areas and was originally developed for medical imaging [12]. This method has been used for enhancement to remove the noise and reduces the edgeshadowing effect in the pre-processing of digital mammogram [13].

Step 1. Mammogram was divided into a number of nonoverlapping contextual regions of equal sizes.

Step 2. The histogram of each contextual region was calculated.

Step 3. A clip limit, for clipping histograms, was set (like $\mathrm{t}=0.001)$.

Step 4. Each histogram was redistributed in such a way that its height did not exceed the clip limit.

Step 5. All histograms were modified by the transformation function

$$
\begin{array}{ll} 
& T\left(r_{k}\right)=\sum_{j=0}^{k} p_{r}\left(r_{j}\right) \\
\text { Where } \quad p_{r}\left(r_{j}\right)=\frac{n_{j}}{n}
\end{array}
$$

Step 6. The neighboring tiles were combined using bilinear interpolation and the gray scale values were altered according to the modified histograms.

In our experiment, we defined tiles size i.e. the rectangular contextual regions to $8 \mathrm{X} 8$, which is chosen from best result from trial. Contrast factor that prevents over-saturation of the image specifically in homogeneous areas is restricted to 0.01 here to get the optimized output. The number of Bins for the histogram building for contrast enhancing transformation is restricted to 64 and the distribution of histogram is 'Rayleigh' or Bell-shaped. The range is not specified in the experiment to get the full range of output image.

\subsection{Binary Homogeneity Enhancement of Digital Mammogram}

In research paper we have proposed a new homogeneity enhancement process namely Binary Homogeneity Enhancement Algorithm (BHEA) for digital mammogram. In this process, each row of mammogram image is treated as an array of data. First step of the process is to determine middle position of array. Value of middle position of array is subtracted from all the element of array starting from first position to the last position of array and check with the maximum difference threshold (MDT) value, which is constant threshold determine by observation. If result of any subtraction is greater than the MDT, the array will be divided into two equal subsets along middle position and the first and last positions of the two subsets will be pushed to stack. Otherwise, the value of middle position will be propagated to all other position after modifying value using uniform color quantization technique in color space breaking in eight level scales. The process will be continued, popping the start and end position subset array from the stack and repeat the aforesaid process. The process will be continued until the stack is empty.

\section{Input: Mammogram image stored in a two dimensional array}

Step 1. Read the next Row of mammogram (initially the first row) to a single dimensional array i.e. Row.

Step 2. Determine the Mid Pixel position of the Row and Read the Intensity of Mid Pixel of the Row.

Step 3. Check the Mid Pixel Intensity with all the pixels stating from First to Last position of the Row.

Step 4. If any Pixel Intensity of the Row is greater than MDT, then divide the Row into two equal parts. If the total positions of Row are odd, then take floor value of Middle position assign to Mid.

Step 5. Push (First and Mid) and (Mid+1 and Last) to stack respectively.

Step 6. If any Pixel Intensity of the Row is not greater than MDT then intensity of Mid pixel will be moved to all the pixels after modifying intensity value using uniform color quantization technique in color space breaking in eight level scales.

Step 7. If the stack is not empty then pop first and last position of Row and go to Step 2.

Step 8. If it is not Last Row go to step 1. 


\subsection{Regions Isolation of Digital Mammogram}

To isolate the different regions of binary homogeneity enhanced mammogram we used seeded region-growing algorithm. It is a procedure that groups pixels or sub regions into larger region based on predefined criteria. The basic approach is to start with a "seed" points and from these grow regions by appending to each seed those neighboring pixels that have properties similar to the seed. This process continues until all pixels are allocated to a region. Growing of a region stops when no more pixels satisfy the criteria for inclusion in that region. We have only taken the border line of each region or segment to compare level of symmetry. Each segment is representing individual portion of human breast in mammogram.

\section{EXPERIMENTAL RESULTS ANALYSIS}

The mammogram images used in this experiment are taken from the mini mammography database of MIAS [14]. In our experiment we have consider all types of breast tissues i.e. Fatty, Fatty-glandular, Dense-glandular and the abnormalities like calcification, well-defined or circumscribed masses, speculated masses and other ill-defined masses. Fifty, medio-lateral oblique (MLO) view of bilateral pairs of mammogram images is used as a test case.

The original mammogram pair and the contrast-enhanced pair are sited in the figure 1 and 2 respectively, in the process over enhancement of noise and reduce the edge-shadowing effect.
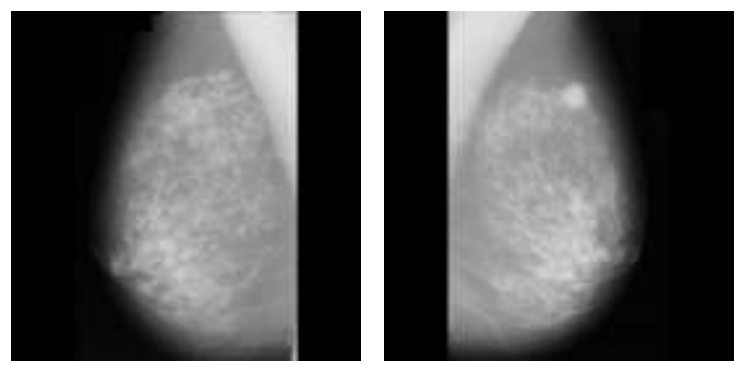

Figure 1. Original mammogram pair before contrast enhancement
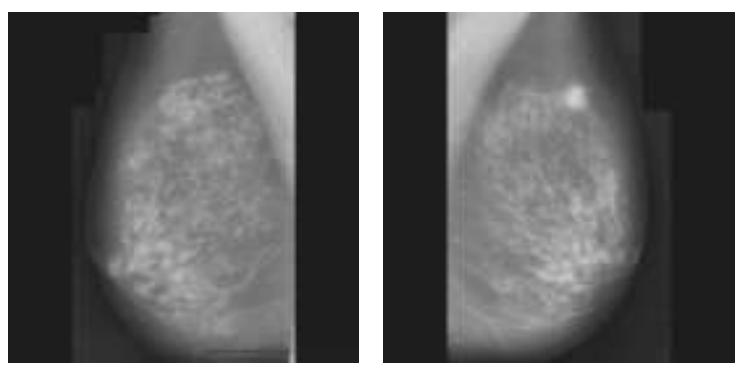

Figure 2. Mammogram pair after contrast enhancement

Binary Homogeneity Enhancement Algorithm (BHEA) is applied on the contrast-enhanced pair, which produces the extraordinary result. Homogeneity of the contrast-enhanced mammogram is increased remarkably along with uniform color quantization technique reduced the color shade to eight in the experiment. The experimental result is depicted in the figure 3 .
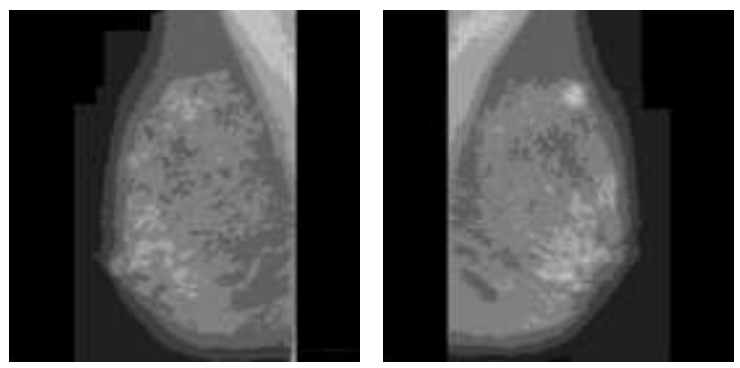

Figure 3. Mammogram pair after using Binary Homogeneity Enhancement Algorithm

Seeded region-growing algorithm is used on the BHEA processed pair where the regions like Pectoral muscles, Lobules, Nipple, Ducts, Fatty tissue and Skin are highly segmented to each other. To compare the anatomical symmetry of the mammogram pair and identify the abnormalities, only boarder of the region or segment is extracted. The exaction of mammogram pair is sited in the figure 4 to authenticate experimental result; right breast image is flipped horizontally so that the pair has the same orientation thereby making the comparison process simpler.
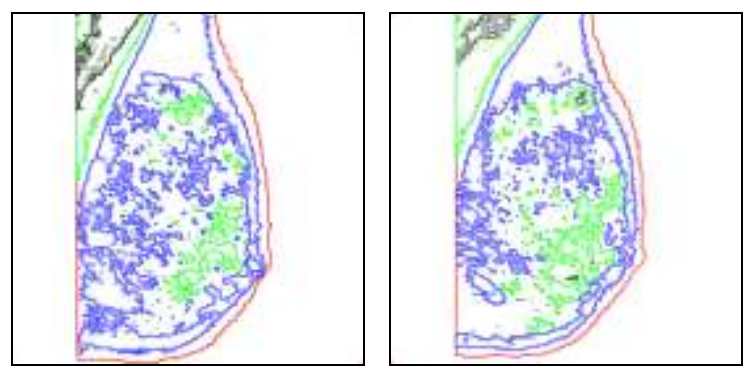

Figure 4. Mammogram pair after extraction of regions

\section{DISCUSSION}

The findings of experimental result shows that using proposed method we can derive the anatomical asymmetry very clearly in breast pair. In figure 4 asymmetry of breast boarder, nipple position is identified very clearly; on the other hand, anatomical disproportion is also visible in terms of lobules, fatty tissues, pectoral muscle and ducts. The presence of abnormal mass is also noticed in the final outcome described in figure 5 . The research work may use for abnormal mass detection, symmetry analysis of breast pair, both cosmetic and reconstruction surgery etc. The level of asymmetry can be determined from our research work by fusing the pair. Importance of the work is high due to the level of asymmetry can predict the degree future risk and remedial process in advance. 


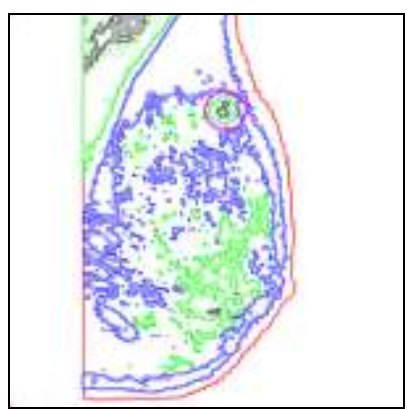

Figure 5. Mammogram showing presence of abnormal mass

\section{CONCLUSION}

In the proposed method, we have Processed Mammogram by contrast-enhanced, BHEA and seeded region growing algorithm to produce the final result. The proposed method has been implemented by $\mathrm{C}$ language and different outputs of the functions are given in the paper. The proposed technique, we have obtained $90 \%$ of near accurate result including accurate results and has the potential for further development. In future work, we plan to make a thorough comparative analysis of the use of different fusion methods in digital mammography to develop a new method to identify the bilateral asymmetry based on correlation between the different regions or segments of interest in mammographic images.

\section{REFERENCE}

[1] Story from BBC NEWS, http://news.bbc.co.uk/go/pr/fr//2/hi/health/4817290.stm, Published on 2006/03/19.

[2] National Statistics UK OnLine http://www.statistics.gov.uk/ cci/nugget.asp?id=575

[3] F. Georgsson, "Differential analysis of bilateral mammograms", International Journal on Pattern Recognition and Artificial Intelligence, Vol. 17, July 2003, pp. 12071226

[4] W.F. Good, X.H. Wang, G. Maitz, "Featurebased Differences Between Mammograms", Medical Imaging
2003: Image Processing, Proceedings of SPIE, Vol. 5032, 2003, pp. 919-929.

[5] A.J. Méndez, P.G. Tahoces, M.J. Lado, M. Souto, J.J. Vidal, "Computer-Aided Diagnosis: Automatic Detection of Malignant Masses in Digitized Mammograms", Medical Physics, Vol. 25, No. 6, June 1998, pp. 957-964

[6] F.F. Yin, M.L. Giger, K. Doi, C.J. Vyborny, R.A. Schmidt, "Computerized Detection of Masses in Digital Mammograms: Automated Alignment of Breast Images and its Effect on Bilateral-Subtraction Technique", Medical Physics, Vol. 21, No. 3, March 1994, pp. 445- 452

[7] Sanmeet Bawa, A thesis on "Edge Based Region Growing", Department of Electronics and communication Engimeering, Thapar Institute of Engineering \& Technology (Deemed University), India, June - 2006

[8] P. J. Besl and R. C. Jain, "Segmentation through variableorder surface fitting," IEEE Trans. Pattern Anal. Machine Intell., vol. PAMI-IO, pp.167-192, 1988

[9] R. M. Haralick and L. G. Shapiro, "Image segmentation techniques," Comput. Vis. Graph. Image Process., vol. 29, pp. 100-132, 1985.

[10] S. L. Horowitz and T. Pavlidis, "Picture segmentation by a directed split-and-merge procedure," Proc. 2nd Int. Joint Conf Pattern Recognit., 1974, pp, 424-433.

[11] Gonzalez, R.C., Woods, R.E. (1992), Digital image processing.

[12] Wanga X, Wong BS, Guan TC, "Image enhancement for radiography inspection", International Conference on Experimental Mechanics, 2004, pp 462-468.

[13] Ball JE, "Digital mammogram spiculated mass detection and spicule segmentation using level sets", Proceedings of the 29th Annual International Conference of the IEEE EMBS, 2007, pp. 4979-4984.

[14] J Suckling et al (1994): The Mammographic Image Analysis Society Digital Mammogram Database Exerpta Medica. International Congress Series 1069 pp 375-378. 